\title{
El receptor NKG2D en la frontera de la inmunovigilancia y la carcinogénesis
}

\author{
Jorge Flavio Mendoza Rincón ${ }^{1}$ DSc
}

1. Unidad de Investigación Multidisciplinaria, Facultad de Estudios Superiores Zaragoza.

Universidad Nacional Autónoma de México.

Correspondencia: mendoza jf@yahoo.com/jflavio.m@gmail.com

Recibido: 11/02/2014 Aceptado: 28/05/2014

\begin{abstract}
Resumen
El sistema inmune es capaz de realizar la detección y eliminación de células transformadas por un mecanismo fisiológico conocido como inmunovigilancia. En este proceso participa el receptor activador NKG2D presente en linfocitos T y células NK, ambos de suma relevancia en la inmunovigilancia contra el cáncer. Al reconocer el receptor NKG2D a sus ligandos (NKG2DLs) en las células que experimentan neotransformación se desencadena la respuesta lítica específica de las células linfoides citotóxicas. Asimismo, se ha descrito en diversos tipos de cáncer formas solubles de NKG2DLs que se ha demostrado son utilizadas para la evasión tumoral al saturar los receptores NKG2D presentes en las células efectoras linfoides evitando de esta manera ser reconocidas y eliminadas y, con ello escapando de la inmunovigilancia. Aunque este fenómeno de evasión inmune, donde participan algunos NKG2DLs, ha sido ya descrito y corroborado clínicamente no se ha estudiado si el receptor NKG2D está presente en las células tumorales per se ya que también podría estar implicado en subvertir la inmunovigilancia. En este trabajo se analizan evidencias recientes de que la expresión del receptor NKG2D no es exclusiva de linfocitos $\mathrm{T}$ y NK ya es expresado por células epiteliales tumorales tanto in vitro como in vivo. Las consecuencias de esta anómala expresión en células no linfoides tiene amplias implicaciones en la carcinogénesis que serán revisadas. También se analizan estudios clínicos recientes donde se comprueba la participación del receptor NKG2D en diferentes patologías tumorales.
\end{abstract}

Palabras clave: carcinogénesis, inmunovigilancia, NKG2D, NKG2DL.

\section{The NKG2D receptor in the border of immune surveillance and carcinogenesis}

\begin{abstract}
The immune system is able to perform the detection and elimination of transformed cells by a mechanism known as physiological immune surveillance. This process involves the NKG2D receptor activator present in T lymphocytes and NK cells, both of paramount importance in the immune surveillance against cancer. To recognize the receptor NKG2D ligands (NKG2DLs) in cells that experience retransformation triggers the specific lithic response of the cytotoxic lymphoid cells. Also, soluble forms of NKG2DLs have been described in various types of cancer that have
\end{abstract}


proven to be used for tumor evasion by saturating the NKG2D receptors present in the effector lymphoid cells thus avoiding their recognition and elimination, which makes them escape immune surveillance. Although this phenomenon of immune evasion, where some NKG2DLs participate, has already been described and corroborated, clinically, it has not been studied whether the receptor NKG2DL is present in the tumor cells per se because it could also be involved in reversing immune surveillance. This paper analyzes recent evidence that the expression of the NKG2D receptor is not lymphocyte $\mathrm{T}$ and NK exclusive it is already expressed by tumor epithelial cells in vitro and in vivo. Consequences of this anomalous expression in non-lymphoid cells have widespread implications in carcinogenesis, which will be revised. Recent clinical studies to prove the participation of NKG2D receptor in several tumor pathologies are analyzed.

Key Words: carcinogenesis, immune surveillance, NKG2D, NKG2DL.

\section{Introducción}

La revisión del tema se organiza en tres apartados. En el primero, se describe las funciones del receptor activador de linfocitos citotóxicos NKG2D; en el segundo, se establece el papel del receptor NKG2D contra tumores; en el tercero, se puntualiza la función del receptor NKG2D como oncoproteína. Finalmente, y considerando la importancia del receptor NKG2D se plantea la posibilidad de utilizar la presencia de NKG2D para diagnóstico, progresión y terapia en diversas condiciones oncológicas.

\section{El receptor activador de linfocitos citotóxicos NKG2D}

El receptor activador de células NK grupo 2, miembro D (NKG2D) pertenece a los receptores transmembranales tipo 2 relacionados a las lectinas expresado como homodímero principalmente en células $\mathrm{T}$ citotóxicas del sistema innato $(\mathrm{NK})$ $(1,2)$ como adaptativo (linfocitos $\left.\mathrm{CD}^{+}\right)(3,4)$. Aunque también se ha demostrado su presencia en linfocitos NKT $(5,6)$, en linfocitos T con receptores $\gamma \delta(7,8)$ y en ciertas subpoblaciones de linfocitos CD4 $(9,10)$ Tabla 1.

Tabla 1. Células del sistema inmune innato y adaptativo expresando el receptor activador NKG2D. Recientemente, ha sido demostrado la presencia de NKG2D en células no linfoides como en líneas de carcinoma de cérvix y de mama. También, se ha corroborado en biopsias de pacientes en varios tumores clínicos.

\begin{tabular}{lll} 
Tipo celular & \multicolumn{1}{c}{ Expresión NKG2D } & \multicolumn{1}{c}{ Función } \\
\hline Células NK & Constitutiva & Inmunovigilancia \\
\hline Linfocitos T & Constitutiva & Citotoxicidad \\
\hline Células NKT & Activación & Inmunovigilancia/Citotoxicidad \\
\hline Linfocitos CD4 & Activación/Proliferación & Activación \\
\hline Linfocitos CD8 & Constitutiva & Activación/Citotoxicidad \\
\hline Macrófagos & Activación/Proliferación & Activación \\
\hline Líneas de cáncer de cérvix & Transformación/Proliferación & Carcinogénesis \\
\hline Líneas de cáncer de mama & Transformación/Proliferación & Carcinogénesis \\
\hline Biopsias pacientes oncológicos & Desarrollo tumoral/Transformación & Aumento masa tumoral/Metástasis \\
\hline
\end{tabular}


El receptor NKG2D reconoce a sus ligandos conocidos genéricamente NKG2DLs algunos emparentados a moléculas de clase-I humanas entre los que se encuentran MICA y MICB $(11,12)$ así como moléculas relacionadas con la proteína viral UL-16 denominadas ULBPs $(13,14)$. Es importante mencionar que los MICs y ULBPs son expresados básicamente en condiciones de estrés fisiológico, esto es en infecciones virales y transformación maligna ya que en condiciones normales su expresión es baja o nula $(15,16)$. Por lo que respecta al gen que codifica al receptor NKG2D se denomina KLRK1 (killer cell lectin-like receptor subfamily member 1, por sus siglas en inglés) y se encuentra localizado en el complejo de genes NK (NKC por sus siglas en inglés) en el cromosoma 12 humano, Figura 1. Tanto MICA, MICB como las ULBP son moléculas expresadas en condiciones de estrés celular y se presentan en la membrana de las células inmunocomprometidas que incluyen tumores de origen epitelial, células leucémicas (17), así como en células infectadas por virus (18), por estrés nutricional (19), hipoxia (20) y daño al ADN (21), entre otras condiciones.

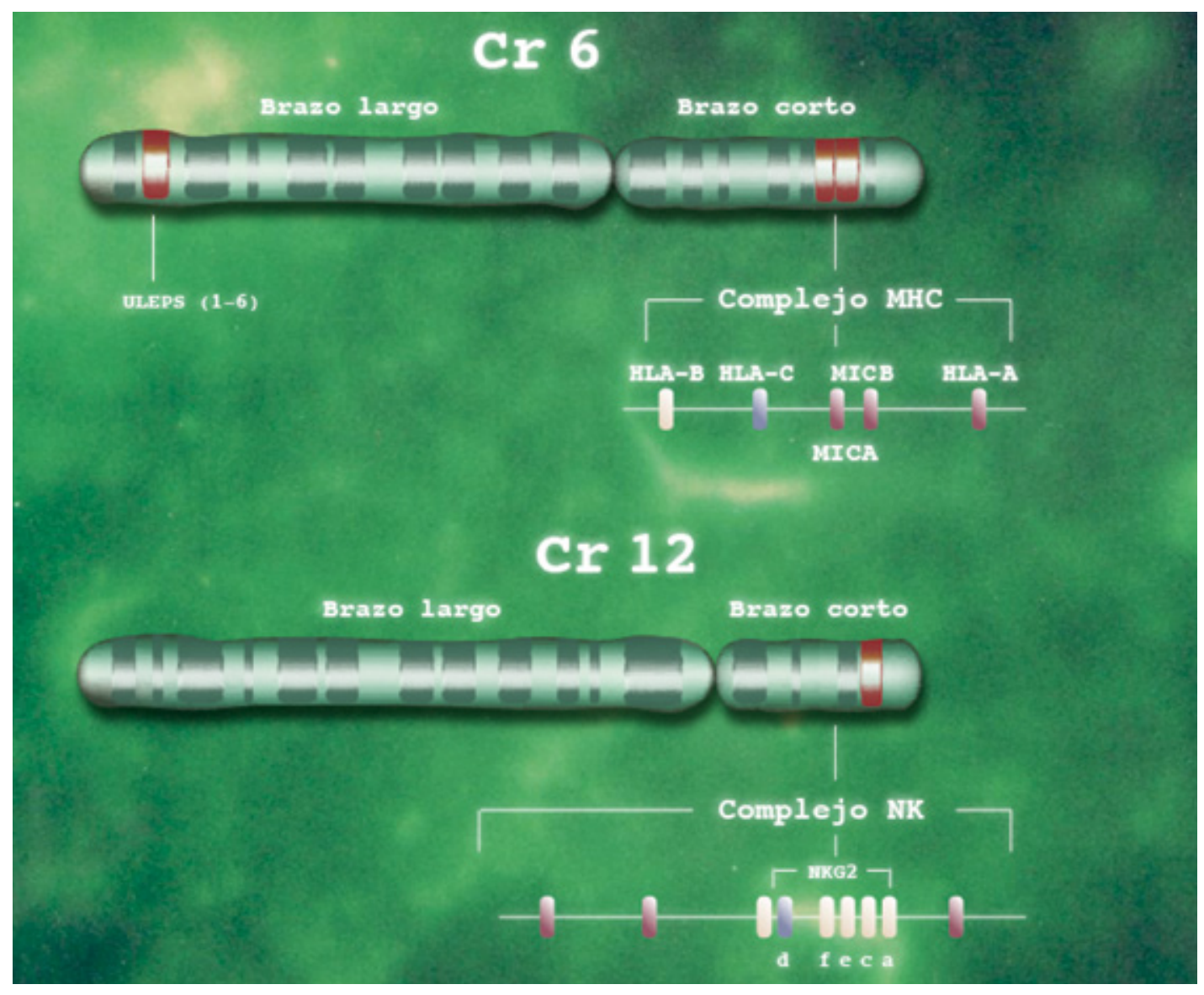

Figura 1. Localización cromosómica de los ligandos MICA, MICB, ULBPs en el cromosoma 6 humano y del receptor NKG2D en el complejo NKG2 cromosoma 12. 
De esta manera, los ligandos NKG2DLs pueden ser reconocidos por el receptor activador NKG2D y con ello desencadenar la actividad lítica característica de las células citotóxicas $\mathrm{T}$ y NK y la subsecuente eliminación de las células bajo estrés y tumorales (22). Adicionalmente, la secreción de MICA, MICB y de ULBPs por varios tipos de células cancerosas se ha sugerido como un mecanismo de escape inmune utilizado por el tumor que provoca la saturación de los receptores NKG2D en las células citotóxicas imposibilitando ser reconocidas y eliminadas por ellas $(23,24)$. De hecho, se ha detectado elevados niveles de MICA, MICB y algunas ULBPs en el suero de pacientes con diversos tipos de cáncer encontrándose una correlación directa entre el incremento de estas moléculas y un mal pronóstico, Tabla 2.

Tabla 2. Presencia de NKG2DL (MICA, MICB y ULBP) en suero de pacientes con diversos padecimientos oncológicos.

sMICA (MICA soluble), sMICB (MICB soluble), sULBP (ULBP soluble).

\begin{tabular}{|lccccc}
\hline Padecimiento oncológico & sMICA & sMICB & sULBP & Referencia \\
\hline Carcinoma hepatocelular & si & & & Zhang et al (ref.41) \\
\hline Melanoma metastásico & si & si & si & Paschen et al (ref.42) \\
\hline Cáncer de ovario & si & si & si & Li et al (ref.43) \\
\hline Leucemia linfocítica crónica células B & & si & Tamaki et al (ref.45) \\
\hline Carcinoma oral de células escamosas & & si & & Salih et al (ref. 46) \\
\hline Cáncer gástrico & si & & Orozco et al (ref.47) \\
\hline Cáncer de vejiga & si & & si & Liu et al (ref. 48) \\
\hline Cáncer de próstata & & si & & Leung et al (ref. 49) \\
\hline Cáncer de colón & si & si & si & He et al (ref. 50)
\end{tabular}

\section{El receptor NKG2D y su función en la inmunovigilancia contra tumores}

En la década de los 50's, Thomas y Burnet desarrollaron la teoría de inmunovigilancia antitumoral $(25,26)$. Establecieron que el sistema inmune se encuentra en estado de alerta antitumoral permanente con capacidad de destruir cuantas células neoplásicas surjan en el organismo (27). Los tumores por tanto serían resultado de un fallo producido en este mecanismo de vigilancia. Aunque en un inicio existió controversia acerca de este proceso fisiológico de inmunovigilancia, la posterior identificación de antígenos tumorales en diversos tipos de neoplasias así como el estudio en modelos animales y ulteriores pruebas clínicas demostraron que el sistema inmune tiene la capacidad de eliminación de cánceres incipientes $(28,29)$. Aquí es importante destacar que los linfocitos $T$ son las cé- lulas por excelencia en este proceso de reconocimiento y eliminación de antígenos tumorales.

Sin embargo, existe otro grupo de células linfoides que no reconocen de manera específica antígenos tumorales pero que tienen la capacidad por igual de identificar y eliminar células transformadas. Este grupo de células linfoides es denominado células NK (Natural Killer, por sus siglas en inglés) y su mecanismo se basa en reconocer diversas señales celulares a través de un balance muy fino donde participan una miríada de receptores inhibidores y activadores que traducen las señales del microambiente $(30,31)$. Es a través de éstos que las células NK pueden identificar a moléculas inducidas durante condiciones de estrés celular o de transformación tumoral reconociendo a los ligandos 
MICA/MICB o ULBPs desencadenando sus funciones citotóxicas características. De hecho, la ausencia del receptor NKG2D en modelos murinos provoca un aumento de la masa tumoral e incremento de la metástasis en carcinomas y leucemias lo que pone en evidencia su importancia en la inmunovigilancia tumoral $(32,33)$.

\section{El receptor NKG2D y su función como oncoproteína}

Nuestro grupo de trabajo ha demostrado en dos líneas de cáncer de cérvix humano denominadas CALO e INBL la expresión del receptor NKG2D lo que constituye el primer reporte de su presencia en células epiteliales tumorales de este receptor activador que se pensaba exclusivo de células linfoides (34). Este hecho, por si mismo resultó muy interesante de descifrar ya que aunque había sido descrito que diversos tipos de tumores expresaban y secretaban los ligandos para este receptor (MICA/MICB/ULBPs) no se sabía que células epiteliales podían expresar de manera simultánea el receptor NKG2D exclusivo de linfocitos T y NK. Dado este fenómeno y de que se desconocía el efecto biológico de MICA/MICB en las células tumorales mismas supusimos que podrían estar relacionadas con su proliferación (34).

Efectivamente, se encontró una estimulación importante de la proliferación en las líneas de cáncer cervical y en varias líneas de leucemia humana. La presencia del receptor NKG2D no es exclusiva de células linfoides sino además está presente en células epiteliales tumorales, hecho que puede estar asociado a un aumento en la tasa de proliferación de estas células tumorales evitando por un lado ser reconocidas y eliminadas por las células efectoras y, por el otro, utilizar estos receptores activadores para incrementar la masa tumoral, la angiogénesis, extravasación y su metástasis evadiendo por un lado la inmunovigilancia y utilizando estos elementos como un mecanismo de invasividad y metástasis por el otro $(35,36)$. Asimismo, resultados recientes han permitido corroborar que este fenómeno es más generalizado en células epiteliales tumorales ya que también hemos detectado la expresión de NKG2D en líneas de cáncer de mama (resultados no publicados).

En estudios clínicos recientes, diversos tipos de tumores expresan de manera importante este receptor incluso se ha vinculado esta presencia a mal pronóstico por un incremento de metástasis en los pacientes portadores debido a un aumento de la vascularización del tumor $(37,38)$. Tomando en consideración lo anteriormente señalado, la expresión del receptor NKG2D se está considerado un blanco importante para inmunoterapia y podría ser útil como biomarcador entre otros ya que su presencia está asociado a etapas iniciales de tumorogénesis así como un incremento de la invasividad y metástasis $(39,40)$, Figura 2.

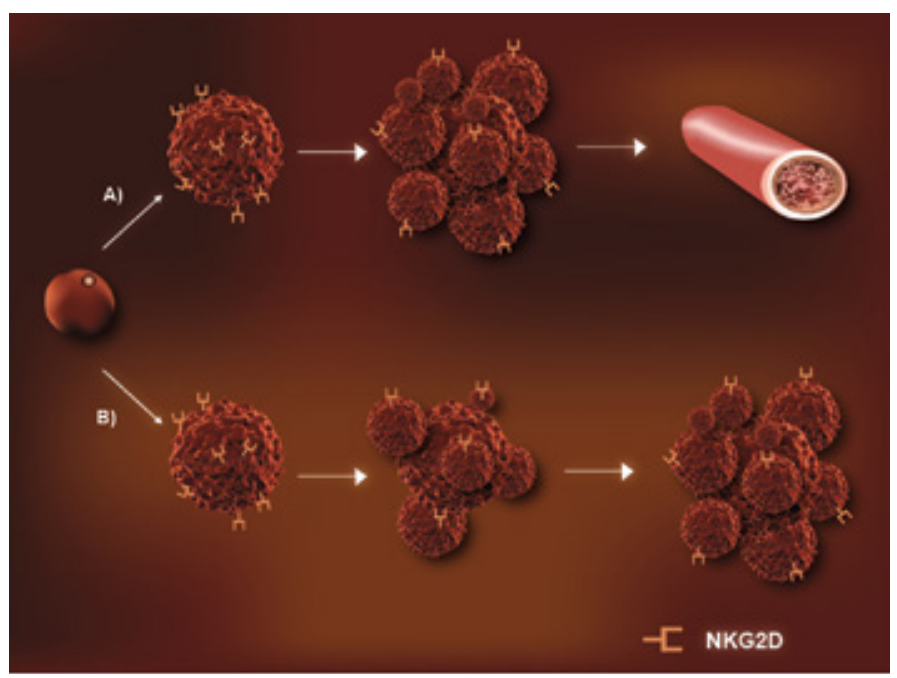

Figura 2. La expresión del receptor NKG2D favorece la carcinogénesis. La presencia de NKG2D favorece el aumento en la metástasis (A) y el aumento de la masa del tumor (B). 


\section{Perspectivas y conclusiones}

La inmunovigilancia es un mecanismo fisiológico cuya finalidad es proteger al organismo de la carcinogénesis y de varias situaciones externas e internas que comprometen el status quo. Las células del sistema inmune innato y adaptativo poseen receptores específicos como NKG2D que reconocen, interpretan y traducen eventos neoplásicos emergentes y diversas infecciones con la finalidad de eliminar estas amenazas. Sin embargo, las células tumorales han desarrollado estrategias para subvertir este reconocimiento como es el caso del sistema NKG2D/NKG2DL por el que se valen para utilizarlo a su favor.

La demostración del receptor NKG2D en células epiteliales tumorales, además de linfoides, y

\section{Referencias}

1. Yokoyama WM, Plougastel BF. Immune functions encoded by the natural killer gene complex. Nat Rev Immunol. 2003; 3:304316.

2. Yokoyama WM, Seaman WE. The Ly-49 and NKRP1 gene families encoding lectin-like receptors on natural killer cells: the NK gene complex. Annu Rev Immunol. 1993; 11:613-635.

3. Burgess SJ, Maasho K, Masilamani M, Narayanan S, Borrego F, Coligan JD: The NKG2D receptor: immunobiology and clinical implications. Immunol Res 2008; 40:18-34.

4. Jonjic' S, Polic' B, Krmpotic': The role of NKG2D in immunoevasion by tumors and viruses. Eur J Immunol. 2008; 38:29272968.

5. Koch J, Steinle A, Watzl C, Mandelboim O. Activating natural cytotoxicity receptors of natural killer cells in cancer and infection. Trends Immunol. 2013; 34:182-191.

6. Zafirova B, Wensveen FM Gulin M, Polic B. Regulation of immune cell function and differentiation by the NKG2D activating receptor. Cell Mol Life Sci. 2011; 68:3519-3529.

7. Raulet DH, Gasser S, Gowen BG, Deng W, Jung H. Regulation of ligands for the NKG2D activating receptor. Annu Rev Immunol. 2013; 31:313-341.

8. Nausch N, Cerwenka A. NKG2D ligands in tumor immunity. Oncogene. 2008; 27:5944-5958.

9. Wrobel P, Shojaei B, Schittek F, Gieseler B, Wollenberg H, Kalthoff D, Kabelitz D, Wesch D: Lysis of a broad range of epithelial tumour cells by human gammadelta $T$ cells: involvement of NKG2D ligands and T-cell receptor-versus NKG2D-dependent recognition. Scand J Immunol. 2007; 66:320-328.

10. Saez-Borderias A, Guma M, Angulo A, Vellosillo B, Pende D, Lopez-Botet M: Expression and function of NKG2D in $\mathrm{CD}^{+} \mathrm{T}$ cells specific for human cytomegalovirus. Eur J Immunol. 2006; 36:3198-3206.

11. Li P, Morris DL, Willcox BE, Steinle A, Spies T, Strong RK. Com- su consecuente vinculación en el aumento de la masa del tumor y metástasis ha abierto la caja de pandora. Adicionalmente, la identificación clínica de este receptor activador en varios tumores hace pensar de su vinculación más directa en el fenómeno de carcinogénesis. En contrapartida, también abre la posibilidad de utilizar la presencia de NKG2D para diagnóstico, progresión y terapia en diversas condiciones oncológicas.

\section{Agradecimientos}

Se agradece los recursos financieros otorgados por el PAPIIT de la Universidad Nacional Autónoma de México (Proyecto: IN217213). La participación de Itzel Moreno Martínez e Iván Zermeño Rangel en el diseño de las imágenes.

plex structure of the activating immunoreceptor NKG2D and its MHC clas I-like ligand MICA. Nat Immunol. 2001; 2:443-451.

12. Cerwenka a, Lanier LL. NKG2D ligands: unconventional MHC class I-like molecules exploited by virus and cancer. Tissue Antigens. 2003; 61:335-343.

13. Mendoza-Rincon JF: Human MICA and MICB genes: their biological function and relevance to infection and cancer. In Advances in Cancer Research at UNAM. Edited by: Mas-Oliva J, Ninomiya-Alarcon J, Garcia- Carranca A. Mexico City; Manual Moderno. 2007; 127-135.

14. Fernández-Messina L, Reyburn HT, Valés-Gómez M. Human NKG2D-ligands: cell biology strategies to ensure immune recongnition. Front Immunol. 2012; 3:299.

15. Champsaur M, Lanier LL. Effect of NKG2D ligands expression on host immune responses. Immunol Rev. 2010; 235:267-285.

16. Raulet DH, Guerra N. Oncogenic stress sensed by the immune system: role of natural killer cell receptors. Nat Rev Immunol. 2009; 9:568-580.

17. Waldhauer I, Steinle A. NK cells and cancer immunosurveillance. Oncogene. 2008; 27: 5932-5943.

18. Chalupny NJ, Rein-Weston A, Dosch S, Cosman D: Downregulation of the NKG2D ligand MICA by the human cytomegalovirus glycoprotein UL142. Biochem Biophys Res Commun. 2006; 346:175-181.

19. Ullrich E, Koch J, Cerwenka A, Steinle A. New prospects on the NKG2D/NKG2DL system for oncology. OncoImmunol. 2013; 2:10-9

20. Unni AM, Bondar T, Medzhitov R: Intrinsic sensor of oncogenic transformation induces a signal for innate immunosurveillance. Proc Natl Acad Sci USA. 2008; 05:1686-1691.

21. Kato NJ, Tanaka J, Sugita T, Toubai Y, Miura M, Ibata Y, Syono Y, Ota S, Kondo T, Asaka M, Imamura M: Regulation of the expression of MHC class I-related chain A, B (MICA, MICB) 
via chromatin remodeling and its impact on the susceptibility of leukemic cells to the cytotoxicity of NKG2D-expressing cells. Leukemia. 2007; 21:2103-2108.

22. Papazahariadou M, Athanasiadis GI, Papadopoulos E, Symeonidou I, Hatzistilianou M, Castellani ML, Bhattacharya K, Shanmugham LN, Conti P, Frydas S: Involvement of NK cells against tumors and parasites. Int J Biol Markers. 2007; 22:144-153.

23. Salih HR, Holdenrieder S, Steinle A: Soluble NKG2D ligands: prevalence, release and functional impact. Front Biosci. 2008; 4:2041-2045.

24. Paschen A, Sucker A, Hill B, Moll I, Zapatka M, Nguyen XD, Sim GC, Gutmann I, Hassel J, Becker JC, Steinle A, Schadendorf D, Ugurel S: Differential clinical significance of individual NKG2D ligands in melanoma: soluble ULBP2 as an indicator of poor prognosis superior to S100B. Clin Cancer Res 2009; 15:5208-5215.

25. Burnet M. Cancer: a biological approach. III. Viruses associated with neoplastic conditions. IV. Practical applications. Br Med J. $1957 ; 1: 841-847$

26. Burnet FM. The concept of immunological surveillance. Prog Exp Tumor Res. 1970; 13:1-27.

27. Corthay A. Does the immune system naturally protect against cancer? Front Immunol. 2014; 5 (197):1-8.

28. Paggi A, Prevosto C, Zancolli M, Canevalli P, Musso A, Zocchi MR: NKG2D and Natural Cytotoxicity Receptors Are Involved in Natural Killer Cell Interaction with Self-Antigen Presenting Cells and Stromal Cells. Ann N Y Acad Sci. 2007; 1109:47-57.

29. Mistry AR, O'Callaghan CA: Regulation of ligands for the activating receptor NKG2D. Immunology. 2007; 121:439-47.

30. Kasahara M, Yoshida S. Immunogenetics of NKG2D ligand gene family. Immunogenetics. 2012; 64-12: 855-67.

31. Poggi A, Zocchi R. How to exploit stress-related immunity against Hodgkin's lymphoma. OncoImmunol. 2013, 2:12:1-8.

32. Guerra N, Tan YX, Joncker NT, Choy A, Gallardo F, Xiong N, Knoblaugh S, Cado D, Greenber NR, Raulet DH. NKG2Ddeficient mice are defective in tumor surveillance in models of spontaneous malignancy. Immunity. 2008; 28:571-580.

33. Deguine J, Breart B, Lemaítre F, Bousso P. Cutting edge: tumortargeting antibodies enhance NKG2D-mediated NK cell cytotoxicity by stabilizing NK cell-tumor cell interactions. J Immunol. 2012; 15; 189 (12): 5493-5497.

34. Weiss-Steider B, Soto-Cruz I, Martínez-Campos CA, MendozaRincon JF. Expression of MICA, MICB and NKG2D in human leukemic myelomonocytic and cervical cancer cells. J Exp Clin Can Res. 2011; 30:1-8.

35. El-Gazzar A, Cai X, Reeves RS, Dai Z, Caballero-Benitez A, McDonald DL, Vazquez J, Gooley TA, Sale GE, Spies T, Groh $\mathrm{V}$. Effects on tumor development and metastic dissemination by NKG2D lymphocyte receptor expressed on cancer cells. Oncogene. 2013; 435:1-9.

36. Benitez AC, Dai Z, Mann HH, Reeves RS, Margineantu DH, Groh V, Spies T. Expression, signaling proficiency, and stimulatory function of the NKG2D lymphocyte receptor in human cancer cells. Proc Natl Acad Sci USA. 2011; 108:4081-6.

37. El-Gazzar A, Groh V, Spies T. Immunobiology and conflicting roles of the human NKG2D lymphocyte receptor and its ligands in cancer. J Immunol. 2013; 191(4):1509-1515.

38. Nanbakhsh A, Pochon C, Mallavialle A, Amsellem S, Bourhis $\mathrm{JH}$, Choualb S. c-Myc regulates expression of NKG2D ligands ULBP1/2/3 in AML and modulates their susceptibility to NKmediated lysis. Blood. 2014; 123:3585-3595.

39. Vantourout P, Willcox C, Turner A, Swanson CM, Haque Y, Sobolev O, Grigoriadis A, Tutt A, Hayday A. Immunological visibility: postrtranscriptional regulation of human NKG2D ligands by the EGF receptor pathway. Sci Trnasl Med. 2014; 6:231-239.

40. Wu YL, Ding YP, Tanaka Y, Shen LW, Wei CH, Minato N, Zhang W. gd T cells and their potential for immunotherapy. Int J Biol Sci. 2014; 10:119-135.

41. Zhang J, Xu Z, Zhou X, Zhang H, Yang N, Wu Y, Chen Y, Yang G, Ren T. Loss of expression of MHC class I-related chain A (MICA) is a frequent event and predicts poor survivlal in patients with hepatocellular carcinoma. Int. J Clin Exp Pathol. 2014, 15;7(6):3123-31.

42. Paschen A, Baingo J, Schadendorf D. Expression of stress ligands of the immunoreceptor NKG2D in melanoma: Regulation and clinical significance. 2014. European J Cell Biol.93:49-54.

43. Li K, Mandai M, Hamanishi J, Matsumura N, Suzuki A, Yagi H, Yamaguchi K, Baba T, Fujii S, Konishi L. Clinical significance of the NKG2D ligands, MICA/V an ULBP2 in ovarian cancer: high expression of ULBP2 is an indicator of poor prognosis. 2009. Cancer Immunol, Immunother, 58:641-52.

44. Hilpert J, Grosse-Hovest L, Grunebach F, Buechele C, Nuebling T, Raum T, Steinle A, Salih HR. Comprehensive analysis of NKG2D ligand expression and release in leukemia: implications for NGK2D-mediated NK cell responses. 2012. J Immunol, 189(3):1360-71.

45. Tamaki S, Kawakami M, Ishitani A, Kawashima W, Kasuda S, Yamanaka Y, Shimomura H, Imai Y, Nakagawa Y, Hatake K, Kirita T. Soluble MIBC serum levels correlate with disease stage and survival rate in patients with oral squamous cell carcinoma. 2010. Anticancer Res, 30:4097-101.

46. Salih HR, Rammensee HG, Steinle A. Cutting edge: down-regulation of MICA on human tumors by proteolytic shedding. 2002. J Immunol, 169:4098-102.

47. Orozco-Levi M, Ramírez-Sarmiento A, Borchers M, MurtaNascimento C, Maciá F, Casado B, Polo M, Caballero-Benítez A, Diaz-Quijano F, Gelabert A. Lack of MICA expression predicts a worse prognosis in patients with bladder cancer. 2013. Open J Pathol, 3:41-50.

48. Liu G, Lu S, Wang X, Page ST, Higano CS, Plymate SR, Greenberg NM, Sun S, Li Z, Wu JD. Perturbation of NK cell peripheral homeostasis accelerates prostate carcinoma metastasis. J Clin Invest 2013, 123:4410-22.

49. Leung WH, Vong QP, Lin W, Janke L, Chen T, Leung W. Modulation of NKG2D ligand expression and metastasis in tumors by spironolactone via RXRg activation. J Exp Med 2013, 10:2675-92.

50. He S, Yin T, Li D, Gao X, Wan Y, Ma X, Ye T, Guo F, Sun J, Lin $Z$, Wang Y. Enhanced interaction between natural killer cells and lung cancer cells: involvement in gefitinib-mediated immunoregulation. J Trnasl Med 2013, 11:186. 